\title{
Symmetry and the macroscopic dynamics of antiferromagnetic materials in the presence of spin-polarized current
}

\author{
Helen V. Gomonay and Roman V. Kunitsyn \\ National Technical University of Ukraine "KPI" \\ ave Peremogy, 37, 03056, Kyiv, Ukraine \\ Vadim M. Loktev \\ Bogolyubov Institute for Theoretical Physics NAS of Ukraine, \\ Metrologichna str. 14-b, 03143, Kyiv, Ukraine
}

(Dated: November 12, 2018)

\begin{abstract}
Antiferromagnetic (AFM) materials with zero or vanishingly small macroscopic magnetization are nowadays the constituent elements of spintronic devices. However, possibility to use them as active elements that show nontrivial and controllable magnetic dynamics is still discussible. In the present paper we extend the phenomenologic theory [A.F.Andreev, V.I.Marchenko, Sov. Phys. - Uspekhi, 23 (1980), 21] of macroscopic dynamics in AFMs for the cases typical for spin-valve devices. In particular, we consider the solid-like magnetic dynamics of AFMs with strong exchange coupling in the presence of spin-polarized current and give the general expression for the current-induced Rayleigh dissipation function in terms of the rotation vector for different types of AFMs. Basing on the analysis of linearized equations of motion we predict the current-induced spin-reorientation and AFM resonance, and found the values of critical currents in terms of AFMR frequencies and damping constants. The possibility of current-induced spin-diode effect and second-harmonic generation in AFM layer is also shown.
\end{abstract}

PACS numbers: 72.25.-b, 72.25.Mk, 75.50.Ee

Keywords: Spin transfer torque, spin-polarized current, antiferromagnet

\section{INTRODUCTION}

Spin polarized electric current flowing through the nanoscale magnetic multilayers can exert torque on a ferromagnetic (FM) layer. This effect provides a potentially useful method for magnetization switching and is widely used in spintronics (see, e.g., review ${ }^{1}$ and references therein). The phenomenon of spin transferred torque (STT) was first suggested by Berger ${ }^{2}$ and Slonczewski ${ }^{3,4}$ in their seminal papers, based on a rather general argument: conservation of the angular/spin momentum for a system that includes both free (itinerant) electrons and localized moments. Well-known expression for the Slonczewski's STT term, $\mathbf{T}_{\text {STT }} \propto J\left[\mathbf{M} \times\left[\mathbf{M} \times \mathbf{p}_{\text {cur }}\right]\right]$ (where $J$ is an electrical current and $\mathbf{p}_{\text {cur }}$ is the current polarization, notation $\times$ means cross-product) is treated straightforward from the microscopic considerations for a FM with magnetization vector $\mathbf{M}$. On the other hand, there are many systems with the complicated magnetic structure and vanishingly small (or zero) magnetization (like antiferromagnets (AFMs) or spin glasses). So, the question: "Can we observe STT phenomena in these materials and how should we describe their dynamics in the presence of spin-polarized current?" has naturally appeared soon after the reminded papers ${ }^{2-4}$. Recent experiments $^{5-9}$ give an indirect evidence that the spinpolarized current may also influence the state of AFM layer. In addition, the experiments show that the insertion of AFM layer drastically reduces the critical current density of magnetization switching in the standard spin-valves ${ }^{10}$. These phenomena, along with the effect of anisotropic magnetoresistance ${ }^{11-13}$ and high eigen frequencies of the magnetic modes, make AFMs the promising candidates for the use in spintronic devices.

The problem of STT in AFMs has two aspects. The first aspect, considered theoretically by several groups, ${ }^{14-17}$ concerns the ability of AFM materials to polarize the electric current that flows through it. The second, - "inverse" - aspect, related to the above mentioned experiments, concerns the ability of spin-polarized current to influence the state of AFM layer. In the present paper we are concentrated on the second aspect and study the dynamics of AFM layer coupled electrically with the fixed FM layer (polarizer). Our main goal is to develop a general formalism able to describe the magnetic dynamics of AFM layer induced by already polarized spin current.

At present, theoretical models for interpretation of STT phenomena in AFMs are based on microscopic calculations for some model systems, ${ }^{14-16,18}$ on the LandauLifshitz equations for magnetic sublattices ${ }^{19,20}$ added with the Slonczewski's term, continuity equations for two-sublattice $\mathrm{AFMs}^{21}$ or phenomenological approach in the framework of nonequilibrium thermodynamics. ${ }^{17}$ In most of these models the magnetic structure of AFM is considered as a set of magnetic sublattices or, in other words, embedded FM lattices that are coupled by the exchange interaction. On the other hand, according to Andreev and Marchenko ${ }^{22}$, such an approach has some shortcomings: $i$ ) application of the Landau-Lifshitz equations (added with the STT term ${ }^{17,19,20}$ ) for each of magnetic sublattices is "sometimes questionable though gives 
the correct results in most cases" ${ }^{22}$; ii) description of macroscopic (long-wave) dynamics in terms of sublattice magnetizations is redundant for many AFMs (with three and more sublattices).

To overcome an excessive detailing and model assumptions, Andreev and Marchenko ${ }^{22,23}$ have developed the general phenomenological ("hydrodynamic-like") theory for the description of macroscopic magnetic dynamics of the materials with strong exchange coupling between the sublattices. They have shown that any magnetic structure (including multisublattice magnets) can be characterized with the at most three mutually orthogonal magnetic vectors that could be introduced from symmetry considerations only, irrespective to microscopic mechanisms of AFM ordering.

In the present paper we make an attempt to extend such an approach to the systems that are under the action of spin-polarized current. Starting from the spin conservation principle we derive an expression for the Rayleigh dissipation function and corresponding dynamic equations in terms of macroscopic variables that describe solid-like rotation of the magnetic moments ${ }^{24}$ in the presence of the external current-induced forces (torques). From general considerations we show that the effect of spin torque in AFMs can be indeed as strong as in FM materials and analyze some typical features of currentdriving dynamics for AFMs with the collinear, planar and nonplanar ordering.

\section{DYNAMICS OF ANTIFERROMAGNETS WITHIN LAGRANGE FORMALISM}

Hereafter we consider the system (like a fragment of typical spin-valve) which consists of the hard FM, nonmagnetic (NM), and AFM layers (see Fig. 1 a). FM layer works as a polarizer for conduction electrons. We assume the $\mathrm{FM}$ vector $\mathbf{M}_{\mathrm{pol}}$ (and, correspondingly, current polarization $\left.\mathbf{p}_{\text {curr }} \| \mathbf{M}_{\text {pol }}\right)$ to be fixed and unchanged within FM layer. NM layer works as a buffer which excludes the direct magnetic (exchange or dipole-dipole) interaction between FM and AFM layers. Besides, the thickness of NM layer is supposed to be below the spin-diffusion length, in order to keep the extent of current polarization almost constant throughout the layer. AFM layer is supposed to be rather thin, so, that $i$ ) it can be set into motion due to STT that takes place in a thin region near NM/AFM interface and ii) its magnetic structure can be assumed homogeneous enough within the layer (so called macrospin approximation). An electric current flows perpendicular to the film plane in the case of conducting AFM (so called CPP configuration, see Fig. 1 a) and between FM and AFM layers in the case of nonconducting AFM (CIP configuration). AFM layer can be either compensated AFM, or weak FM, or even spin glass. Following the terminology used for spin-valve structures we will refer to AFM layer as a soft one. (a)

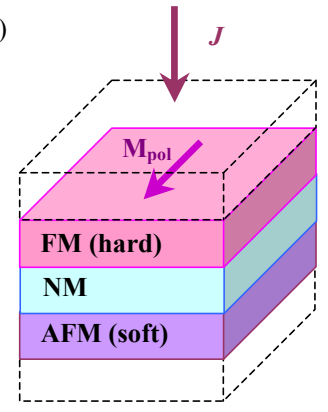

(b)

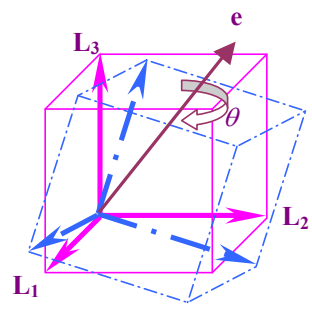

Figure 1. (Color online) Effect of spin transfer torque on AFM layer. (a) Element of spin-valve structure consisting of FM (hard) and AFM (soft) layers and NM spacer. The FM layer polarizes the spin current $\mathbf{J}$ along $\mathbf{M}_{\text {pol }}$ direction. The positive-valued current flows from FM to AFM layer. (b) Macroscopic current-induced dynamics of an arbitrary AFM with strong exchange coupling. Equilibrium magnetic structure is characterized by the three mutually orthogonal magnetic vectors $\mathbf{L}_{1}, \mathbf{L}_{2}$, and $\mathbf{L}_{3}$ (solid arrows). Any deflection from equilibrium (dash arrows) can be viewed as a solid-like rotation around the instantaneous axis e through the angle $\theta$.

\section{A. Comparison of spin transfer torques in ferro- and antiferromagnets}

To clarify the main ideas of the present paper we start from short review of STT phenomenon in ferromagnets where it manifests the spin conservation principle for the system consisting of free (itinerant) and localized spins. ${ }^{25}$

Equations for spin transfer torque in a soft FM can be obtained from the balance equation for the magnetic moment:

$$
\begin{aligned}
& \frac{d \mathbf{M}_{\mathrm{FM}}}{d t}=\nabla \cdot \hat{\boldsymbol{\Pi}}, \\
& \text { or } \quad \frac{d M_{\mathrm{FM}}^{(k)}}{d t}=\frac{\partial \Pi_{k l}}{\partial x_{l}},
\end{aligned}
$$

where $\hat{\boldsymbol{\Pi}}$ is the 2-nd rank tensor of the magnetization flux density induced, in particular, by spin-polarized current. The l.h.s. of Eq. (1) includes the torque $\mathbf{T}_{\mathrm{FM}}$ produced by the effective magnetic field and internal damping (not to be interchanged with the external spin transfer torque). Thus, in the absence of a current the Eq. (1) takes a form of a standard Landau-Lifshits-Gilbert equation:

$$
\begin{aligned}
\frac{d \mathbf{M}_{\mathrm{FM}}}{d t} & \equiv \underbrace{\frac{\partial \mathbf{M}_{\mathrm{FM}}}{\partial t}}_{\mathbf{T}_{\mathrm{FM}}} \\
& +\underbrace{\gamma \mathbf{H}_{\mathrm{ef}} \times \mathbf{M}_{\mathrm{FM}}+\frac{\alpha_{\mathrm{FM}}}{M_{\mathrm{FM}}} \frac{\partial \mathbf{M}_{\mathrm{FM}}}{\partial t} \times \mathbf{M}_{\mathrm{FM}}}_{\mathrm{eff}}=0,
\end{aligned}
$$

where $\gamma$ is the gyromagnetic ratio ${ }^{26}, \mathbf{H}_{\text {eff }}$ is an effective magnetic field, $\alpha_{\mathrm{FM}}$ is the Gilbert damping parameter.

In the macrospin approximation (thin, magnetically homogeneous soft FM layer) the r.h.s. of Eq. (1) can be 
expressed in terms of the magnetization flux (per unit square) transferred by the current $J \equiv j S_{\mathrm{FM}}$ that flows through the FM layer perpendicularly to its surface:

$$
\nabla \cdot \hat{\mathbf{\Pi}}=\frac{\hbar \gamma S_{\mathrm{FM}}}{|e| v_{\mathrm{FM}}} j \hat{\epsilon} \mathbf{p}_{\mathrm{curr}},
$$

where $S_{\mathrm{FM}}$ and $v_{\mathrm{FM}}$ are the surface area and the volume of the FM layer, respectively, $\hbar$ is the Plank constant, $e$ is an electron charge, and, as above, $\mathbf{p}_{\text {curr }}$ is the direction of current polarization, $\left|\mathbf{p}_{\text {curr }}\right|=1$. Second-rank tensor $\hat{\epsilon}$ is proportional to the phenomenological (material) constant $\epsilon_{\mathrm{S}-\mathrm{f}}$ that describes the efficiency of spin-flip scattering of the carriers at the NM/FM interface and depends upon the details of $s d$-exchange interaction between the free and localized electrons. The structure of the tensor in general depends upon the magnetic symmetry of the soft FM layer. In the particular case of isotropic layer ${ }^{4,27}$ (only the exchange symmetry is taken into account)

$$
\hat{\epsilon}=\epsilon_{\mathrm{s}-\mathrm{f}}\left(\hat{1}-\mathbf{e}_{M} \otimes \mathbf{e}_{M}\right), \quad \mathbf{e}_{M} \equiv \frac{\mathbf{M}_{\mathrm{FM}}}{M_{\mathrm{FM}}},
$$

where the symbol $\otimes$ means the direct tensor product, $\hat{1}$ is a unit matrix.

In contrast to ferromagnets, AFMs have complicated, multisublattice magnetic structure and vanishingly small or zero equilibrium macroscopic magnetization. However, the set of dynamic equations for AFMs includes the same spin-flux balance equation (1) as for FMs. The only difference is that in the case of AFMs the vector $\mathbf{M}_{\mathrm{AFM}}$ of macroscopic magnetization is induced by anisotropic Dzyaloshinskii-Moria exchange interactions, by the external magnetic field and, what is essential, by the solid-like rotation of the magnetic lattice, as was shown by Bar'yakhtar and Ivanov ${ }^{28}$ by the direct analysis of the Landau-Lifshitz equations and in Ref. 22 basing on Noether's theorem. For example, in the collinear compensated AFM with an AFM vector $\mathbf{L}$ the macroscopic magnetization $\mathbf{M}_{\mathrm{AFM}} \propto[\mathbf{L} \times \dot{\mathbf{L}}]$.

It follows from the balance equation (1) that the spinflux transferred by the current to AFM layer gives rise to variation of macroscopic magnetization and, as will be shown below, induces rotation of the magnetic lattice. R.h.s. of Eq. (1) is thus can be considered as the Slonczewski's term for STT. In the case of thin AFM layer it can be expressed in a form Eq. (3) with an obvious substitution of $\mathbf{M}_{\mathrm{FM}} \rightarrow \mathbf{M}_{\mathrm{AFM}}, S_{\mathrm{FM}} \rightarrow S_{\mathrm{AFM}}, v_{\mathrm{FM}} \rightarrow v_{\mathrm{AFM}}$. Tensor $\hat{\epsilon}$ has the symmetry-imposed structure analogous to that given by Eq.(4). In the simplest case of spin glasses and some noncollinear AFMs (like FeMn) this tensor is isotropic, some other particular cases will be discussed below.

It should be noted that the spin-polarized current affects an AFM layer in three ways. First of all, due to the flip of the free electron spins at the NM/AFM interface (or within an AFM volume, if the thickness of layer is rather small) the current transfers spin moment and, correspondingly, magnetization to the localized magnetic lattice (see Eq.(3)). This effect is analogous to the Slonczewski's nonadiabatic STT.

Second, the rest of the current that hasn't change polarization, contributes into the effective magnetic field due to exchange coupling (effective constant $I_{\mathrm{sd}}$ ) between the localized and free electrons:

$$
\mathbf{H}_{\text {eff }}^{\text {curr }} \propto \hbar \gamma I_{\text {sd }} j\left(1-2 \epsilon_{\mathrm{s}-\mathrm{f}}\right) \mathbf{p}_{\text {curr }}
$$

(similar to adiabatic spin torque that acts on the FM domain walls, see, e.g. Ref. 29).

At last, the current itself (no matter polarized or no) produces an Oersted field. The last contribution can be easily taken into account in the dynamic equations as a component of the external magnetic field.

The first contribution depends mainly from the scattering properties of the NM/AFM interface and can be equally strong (or equally weak) in FMs and AFMs, independently from the magnetic structure of the soft layer (at least, in the first approximation). The second contribution can play an important role in the case of an ac current and possibly triggers the spin oscillations, in analogy with the effective magnetic field generated by an inverse Faraday effect. ${ }^{30,31}$

\section{B. Dynamic equations}

In what follows we consider the case of AFMs and other multisublattice magnets with strong inter- and intrasublattice exchange couplings - so strong, that the applied magnetic field and/or high density current do not influence the mutual orientation of sublattice magnetizations. The dynamics of such a system can be effectively described as a 3D solid-like rotation of sublattice magnetizations stocked together by the exchange interactions or, equivalently, of one, two, or three mutually orthogonal vectors that characterize the magnetic structure (see Fig. 1 b). Appropriate and adequate technique for description of dynamics of the magnetic structure is the Lagrange formalism which makes it possible to exclude from consideration those degrees of freedoms that are related with the mutual tilt of sublattice magnetizations (so called exchange modes). Convenient parametrization and explicit form of the Lagrange function depends upon the type of magnetic ordering. In noncollinear magnets the $3 \mathrm{D}$ rotation can be parametrized with the help of the Gibbs' vector $\varphi=\varphi \mathbf{e}$, where the unit vector $\mathbf{e}$ defines an instantaneous rotation axis, $\varphi=\tan (\theta / 2)$, and $\theta$ is the rotation angle (see Fig.1 b). Generalized coordinates are generated by the infinitesimal spin rotation $\delta \boldsymbol{\theta}$ (see Appendix A) that represents the difference between rotations $\boldsymbol{\varphi}+d \boldsymbol{\varphi}$ and $\boldsymbol{\varphi}$ :

$$
\delta \boldsymbol{\theta}=2 \frac{d \varphi+\varphi \times d \varphi}{1+\varphi^{2}}
$$

or

$$
\delta \theta_{\alpha}=2 \lambda_{\alpha \beta} d \varphi_{\beta}, \quad \lambda_{\alpha \beta}=\frac{\delta_{\alpha \beta}+\varepsilon_{\alpha \gamma \beta} \varphi_{\gamma}}{1+\varphi^{2}},
$$


where $\varepsilon_{\alpha \gamma \beta}$ is the (completely antisymmetric) Levi-Civita symbol. The components of spin rotation frequency $\boldsymbol{\Omega} \equiv$ $\dot{\boldsymbol{\theta}}$ form a set of corresponding generalized velocities and could be expressed through $\varphi$ as follows:

$$
\Omega=2 \frac{\dot{\varphi}+\varphi \times \dot{\varphi}}{1+\varphi^{2}}
$$

In the presence of the external magnetic field $\mathbf{H}$ the Lagrange function for AFMs takes the form ${ }^{22}$

$\mathcal{L}_{\mathrm{AFM}}=\frac{1}{2 \gamma^{2}} \chi_{\alpha \beta}(\boldsymbol{\varphi})\left(\Omega_{\alpha}+\gamma H_{\alpha}\right)\left(\Omega_{\beta}+\gamma H_{\beta}\right)-U_{\mathrm{AFM}}(\boldsymbol{\varphi})$, where $\chi(\varphi)$ is the magnetic susceptibility tensor that accounts for the exchange symmetry of particular AFM. For the sake of simplicity we consider further the case of the isotropic media (typical for spin glasses and some noncollinear AFMs) with $\chi_{\alpha \beta}(\varphi) \equiv \chi \delta_{\alpha \beta}, \chi=$ const; generalization on more complicated cases is straightforward. The symbol $U_{\mathrm{AFM}}(\boldsymbol{\varphi})$ is the potential energy that depends upon the magnetic anisotropy of the soft layer (see Appendix A).

In the absence of dissipation (and, in particular, spinpolarized current) the dynamic equations are deduced from (9) as Euler-Lagrange equations

$$
\frac{d}{d t}\left(\frac{\partial \mathcal{L}_{\mathrm{AFM}}}{\partial \dot{\varphi}}\right)-\frac{\partial \mathcal{L}_{\mathrm{AFM}}}{\partial \varphi}=0
$$

and take a form:

$$
\frac{2 \chi}{\gamma^{2}} \lambda_{\beta \alpha} \frac{d}{d t}\left[\left(\Omega_{\beta}+\gamma H_{\beta}\right)\right]+\frac{2}{1+\varphi^{2}}\left\{(\mathbf{H} \times \dot{\varphi})_{\alpha}+H_{\beta}\left[\lambda_{\beta \gamma} \dot{\varphi}_{\gamma} \varphi_{\alpha}-\lambda_{\beta \alpha}(\varphi \dot{\varphi})\right]\right\}+\frac{\partial U_{\mathrm{AFM}}}{\partial \varphi_{\alpha}}=0 .
$$

On the other hand, according to Ref. 22, macroscopic magnetization is proportional to spin rotation frequency:

$$
\mathbf{M}_{\mathrm{AFM}}=\frac{\chi}{\gamma}(\boldsymbol{\Omega}+\gamma \mathbf{H})
$$

So, multiplying Eq. (11) by $\gamma \lambda_{\alpha \delta}^{-1}$ one can reduce it to a form

$$
\dot{\mathbf{M}}_{\mathrm{AFM}}+\chi \mathbf{H} \times \boldsymbol{\Omega}+\frac{\gamma}{2} \hat{\lambda}^{-1} \cdot \frac{\partial U_{\mathrm{AFM}}}{\partial \varphi}=0
$$

or, equivalently, with account of Eq. (12):

$$
\begin{gathered}
\frac{\partial \mathbf{M}_{\mathrm{AFM}}}{\partial t}+\underbrace{\gamma \mathbf{H} \times \mathbf{M}_{\mathrm{AFM}}+\frac{\gamma}{2} \hat{\lambda}^{-1} \cdot \frac{\partial U_{\mathrm{AFM}}}{\partial \varphi}}_{\mathbf{T}_{\mathrm{AFM}}}=0 . \quad \text { (14) } \quad \begin{array}{l}
\text { the latter by } 2 \hat{\lambda} / \gamma \text { and taking into account Eq. (3) we } \\
\text { arrive to }
\end{array} \\
\frac{2 \chi \lambda_{\beta \alpha}}{\gamma^{2}} \frac{d}{d t}\left[\left(\Omega_{\beta}+\gamma H_{\beta}\right)\right]+\frac{2}{1+\varphi^{2}}\left\{(\mathbf{H} \times \dot{\varphi})_{\alpha}+H_{\beta}\left[\lambda_{\beta \gamma} \dot{\varphi}_{\gamma} \varphi_{\alpha}-\lambda_{\beta \alpha}(\varphi \dot{\varphi})\right]\right\}+\frac{\partial U_{\mathrm{AFM}}}{\partial \varphi_{\alpha}}=\frac{2}{\gamma} \lambda_{\beta \alpha} g j p_{\text {curr }}^{\beta}, \quad \text { (16) }
\end{gathered}
$$

Here $\lambda_{\alpha \beta}^{-1}=\delta_{\alpha \beta}+\varepsilon_{\alpha \beta \gamma} \varphi_{\gamma}+\varphi_{\alpha} \varphi_{\beta}$ is the tensor inverse to $\lambda_{\alpha \beta}$. Partial time derivative is used to stress that the free variables, in addition to time, include the generalized coordinated $\varphi$.

It can be easily seen that Eq. (14) looks similar to Eq. (2). The torque $\mathbf{T}_{\mathrm{AFM}}$ is produced by the external field (like in FMs) and by the magnetic anisotropy (similar but not equal to that in FMs). Thus, equations (13), (14) and hence (11) could be treated as the balance equation for magnetization which in the absence of current has a form ${ }^{32}$

$$
\frac{d \mathbf{M}_{\mathrm{AFM}}}{d t}=0 .
$$

In the presence of spin-polarized current the balance equation (15) transforms into Eq. (1). Then, multiplying where we have introduced the phenomenological constant $g \equiv \hbar \gamma S_{\mathrm{AFM}} \epsilon_{\mathrm{s}-\mathrm{f}} /\left(|e| v_{\mathrm{AFM}}\right)$.

Eq. (16) can be considered as the Lagrange equation in the presence of the external dissipative forces

$$
\frac{d}{d t}\left(\frac{\partial \mathcal{L}_{\mathrm{AFM}}}{\partial \dot{\varphi}}\right)-\frac{\partial \mathcal{L}_{\mathrm{AFM}}}{\partial \varphi}=-\frac{\partial \mathcal{R}_{\mathrm{AFM}}}{\partial \dot{\varphi}}
$$

where $\mathcal{R}_{\mathrm{AFM}}$ is the Rayleigh dissipation function, $\mathcal{R}_{\mathrm{AFM}}$ is related with the rate of the energy $\mathcal{E}_{\mathrm{AFM}}$ losses as follows:

$$
\frac{d \mathcal{E}_{\mathrm{AFM}}}{d t} \equiv-\dot{\varphi}\left(\frac{\partial \mathcal{R}_{\mathrm{AFM}}}{\partial \dot{\varphi}}\right) .
$$

Comparison of Eqs. (17) and (16) with account of expressions (7) and (8) shows that the current-induced contribution into dissipation function can be presented as 


$$
-g j\left(\boldsymbol{\Omega} \cdot \mathbf{p}_{\text {curr }}\right) / \gamma
$$

The internal losses (Gilbert damping) in AFM layer is taken into account in a standard way. As a result, dissipation function takes a form

$$
\mathcal{R}_{\mathrm{AFM}}=\frac{\alpha_{\mathrm{AFM}}}{2 \gamma} \boldsymbol{\Omega}^{2}-\frac{g j}{\gamma}\left(\boldsymbol{\Omega} \cdot \mathbf{p}_{\mathrm{curr}}\right),
$$

where $\alpha_{\mathrm{AFM}}$ is a damping constant of AFM layer.

Expression (19) can be written in terms of the Gibbs vector as follows (see Eq. (8)):

$$
\mathcal{R}_{\mathrm{AFM}}=\frac{2 \alpha_{\mathrm{AFM}}}{\gamma}\left[\frac{\dot{\varphi}^{2}}{1+\varphi^{2}}-\frac{(\boldsymbol{\varphi} \cdot \dot{\varphi})^{2}}{\left(1+\varphi^{2}\right)^{2}}\right]-\frac{2 g j}{\gamma} \frac{\mathbf{p}_{\mathrm{curr}} \cdot \dot{\varphi}+\mathbf{p}_{\mathrm{curr}} \cdot(\boldsymbol{\varphi} \times \dot{\varphi})}{1+\varphi^{2}}
$$

The expression (20) for the Rayleigh function is the main result of the present paper. Together with the Lagrange function (9) it describes the dynamics of AFMs with strong exchange coupling in the presence of spinpolarized current.

As it was already shown, expressions (19), (20) for the Rayleigh function result from the spin conservation principle and thus are rather general. However, the analogous expressions could be deduced directly from the LandauLifshits-Slonczewski equations for the magnetic sublattices with the account of strong exchange coupling between them. ${ }^{20}$ Moreover, for the case of FMs the currentinduced contribution into the Rayleigh function has a similar form (see Ref. 33):

$$
\mathcal{R}_{\mathrm{FM}}=\ldots-\frac{g j}{\gamma M_{0}^{2}} \mathbf{p}_{\mathrm{curr}} \cdot(\mathbf{M} \times \dot{\mathbf{M}})
$$

where by ... we denote the contribution from the internal damping.

Analysis of the expression (19) shows that the stationary state of AFMs (with $d \mathcal{E}_{\mathrm{AFM}} / d t=0$ ) in the presence of steady spin-polarized current corresponds to rotation of the magnetic structure around the current polarization $\mathbf{p}_{\text {curr }}$ with the constant frequency

$$
\boldsymbol{\Omega}=\frac{g}{2 \alpha_{\mathrm{AFM}}} j \mathbf{p}_{\mathrm{curr}}
$$

The rotation frequency can be controlled by the current value $j$ and depends upon the loss factor $\alpha_{\mathrm{AFM}}$.

\section{DISSIPATION FUNCTION FOR DIFFERENT TYPES OF ANTIFERROMAGNETS}

The method described in the Sec.II can be easily generalized to the systems of different exchange symmetry. In this section we analyze some typical cases useful for applications. For the sake of clarity we reproduce the expressions for the kinetic energy from Ref. 22 as well.

\section{A. Noncollinear antiferromagnets and disordered magnets}

We start from the simplest case of noncollinear AFMs with the cubic exchange symmetry. A typical example is given by the metallic AFM FeMn widely used in spin-valves. Magnetic structure of FeMn (see Fig. 2 a,b) consists of four equivalent magnetic sublattices $\mathbf{M}_{k}$ $(k=1, \ldots, 4)$ oriented along four $\langle 111\rangle$ directions of cubic cell. ${ }^{34}$ Macroscopic magnetization $\left(\mathbf{M}_{\mathrm{AFM}}=\sum \mathbf{M}_{k}\right)$ of FeMn in equilibrium is zero, so, the magnetic structure is described by three orthogonal AFM vectors that could be related with the sublattice magnetizations as follows:

$$
\begin{aligned}
& \mathbf{L}_{1}=\mathbf{M}_{1}+\mathbf{M}_{2}-\mathbf{M}_{3}-\mathbf{M}_{4}, \\
& \mathbf{L}_{2}=-\mathbf{M}_{1}+\mathbf{M}_{2}-\mathbf{M}_{3}+\mathbf{M}_{4}, \\
& \mathbf{L}_{3}=\mathbf{M}_{1}-\mathbf{M}_{2}-\mathbf{M}_{3}+\mathbf{M}_{4}
\end{aligned}
$$

It is interesting that such a complicated structure is predicted not only in the bulk material, but also in the thin (down to $1.5 \mathrm{~nm}^{35,36}$ ) FeMn layer within multilayered structures used in spintronic devices. ${ }^{6,37}$

All three vectors $\mathbf{L}_{k}$ have the same modulus, so, magnetic structure in the exchange approximation has a cubic symmetry and tensors $\hat{\epsilon}$ and $\hat{\chi}$ are isotropic. Thus, the Rayleigh function is given by the Exp. (20) and the kinetic energy has a form:

$T_{\text {kin }}=\frac{2 \chi}{\gamma^{2}}\left[\frac{\dot{\varphi}^{2}}{1+\varphi^{2}}-\frac{(\boldsymbol{\varphi} \cdot \dot{\varphi})^{2}}{\left(1+\varphi^{2}\right)^{2}}\right]+\frac{2 \chi}{\gamma} \frac{\mathbf{H} \cdot \dot{\varphi}+\mathbf{H} \cdot(\boldsymbol{\varphi} \times \dot{\varphi})}{1+\varphi^{2}}$.

Similar expressions for the kinetic energy and Rayleigh function are obtained for the disordered systems that according to Ref. 22 include spin glasses (no macroscopic magnetization) and disordered $\mathrm{AFMs}^{38}$ (zero macroscopic magnetization and nonzero AFM vectors). These systems show strong magnetic correlations induced by exchange coupling between the nearest localized spins and are isotropic with respect to any spin rotations. 
(a)

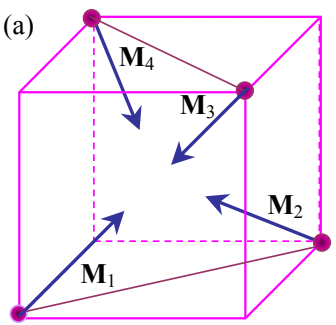

(b)

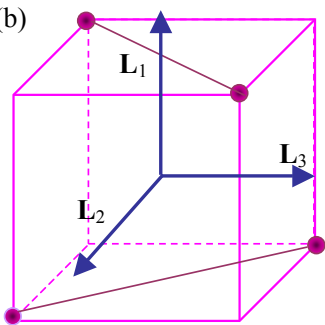

(c)

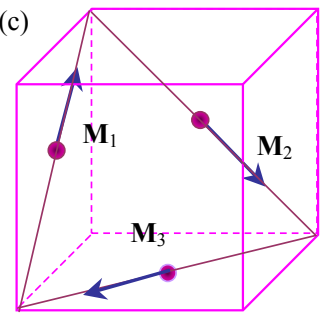

(d)

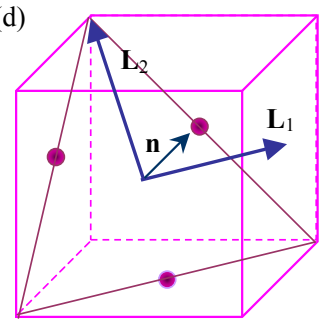

Figure 2. (Color online) Magnetic structure of the cubic noncollinear AFMs. (a) Magnetic structure of the disordered FeMn. Magnetic atoms (circles) form fcc lattice, vectors $\mathbf{M}_{k}$ of sublattice magnetizations (arrows) point to $\langle 111\rangle$ directions. (b) This structure can be equivalently described in terms of three mutually orthogonal AFM vectors $\mathbf{L}_{k}$ (see Eq. (23)). (c) Planar magnetic structure of $\mathrm{IrMn}_{3}$ and some of the antiperovskites $\mathrm{Mn}_{3} \mathrm{MN}$, where $\mathrm{M}=\mathrm{Ni}, \mathrm{Ga}, \mathrm{Ag}, \mathrm{Zn}$. Magnetic moments $\mathbf{M}_{k}$ (arrows) are localized on Mn atoms (circles). (d) Planar magnetic structure can be described by two mutually orthogonal AFM vectors $\mathbf{L}_{k}$ (see Eq. (25)). The plane of AFM ordering is described by the unit vector $\mathbf{n} \| \mathbf{L}_{1} \times \mathbf{L}_{2}$.

\section{B. Planar noncollinear antiferromagnets}

Noncollinear AMFs with triangular magnetic structure (see Fig. 2c,d) are interesting and important for applications. In particular, such type of structure is observed in $\operatorname{IrMn}_{3}$ alloy ${ }^{39}$ which is, as well as FeMn, used as a pinning layer in spintronic devices (see, e.g., Ref.40 and 41 ), and in the series of metallic antiperovskites $\mathrm{Mn}_{3} \mathrm{MN}$ (where $\mathrm{M}=\mathrm{Ni}, \mathrm{Ga}, \mathrm{Ag}, \mathrm{Zn})^{42}$ used in composite materials due to strong negative thermal expansion (see, e.g. Refs. 43-45). Some of the antiperovskites also show giant magnetoresistance effect ${ }^{46}$ in AFM phase and thus can be susceptible to the action of spin-polarized current.

Magnetic structure of cubic $\mathrm{IrMn}_{3}$ and $\mathrm{Mn}_{3} \mathrm{MN}$ is represented by three equivalent magnetic sublattices with magnetization vectors $\mathbf{M}_{k},(k=1,2,3)$ that make $120^{\circ}$ angle with respect to each other. Two mutually orthogonal AFM vectors,

$$
\mathbf{L}_{1}=\mathbf{M}_{1}+\mathbf{M}_{2}-2 \mathbf{M}_{3}, \quad \mathbf{L}_{2}=\sqrt{3}\left(\mathbf{M}_{1}-\mathbf{M}_{2}\right)
$$

define the plane of the magnetic ordering (in exchange approximation) with the normal vector $\mathbf{n} \| \mathbf{L}_{1} \times \mathbf{L}_{2}$, and equilibrium magnetization $\mathbf{M}_{\mathrm{AFM}}=\sum \mathbf{M}_{k}=0$.

Exchange symmetry group is isomorphous to $C_{3 h}$, where rotation axis is parallel to $\mathbf{n}$, so, any 2-nd rank

symmetric tensor has two independent components - parallel and perpendicular to $\mathbf{n}$ direction. For example, tensor of magnetic susceptibility takes a form:

$$
\hat{\chi}=\chi_{\perp} \hat{1}+\left(\chi_{\|}-\chi_{\perp}\right) \mathbf{n} \otimes \mathbf{n} .
$$

As a result, the kinetic energy and Rayleigh function can be written as:

$$
\begin{aligned}
T_{\text {kin }} & =\frac{\chi_{\perp}}{2 \gamma^{2}}(\boldsymbol{\Omega}+\gamma \mathbf{H})^{2}+\frac{\chi_{\|}-\chi_{\perp}}{2 \gamma^{2}}[(\boldsymbol{\Omega}+\gamma \mathbf{H}) \cdot \mathbf{n}]^{2}, \\
\mathcal{R}_{\mathrm{AFM}} & =\frac{\alpha_{\perp}}{2 \gamma} \boldsymbol{\Omega}^{2}+\frac{\alpha_{\|}-\alpha_{\perp}}{2 \gamma}(\boldsymbol{\Omega} \cdot \mathbf{n})^{2}-\frac{g_{\perp} j}{\gamma}\left(\boldsymbol{\Omega} \cdot \mathbf{p}_{\mathrm{curr}}\right) \\
& -\frac{\left(g_{\|}-g_{\perp}\right) j}{\gamma}(\boldsymbol{\Omega} \cdot \mathbf{n})\left(\mathbf{n} \cdot \mathbf{p}_{\mathrm{curr}}\right) .
\end{aligned}
$$

The functions $T_{\text {kin }}$ and $\mathcal{R}_{\mathrm{AFM}}$ from the above expression could be easily expressed in terms of the Gibbs' vector using the relation (8).

\section{Collinear antiferromagnets}

The collinear AFMs with high Neel temperature, like IrMn and $\mathrm{NiO}$, are also widely used in spin-valves. Their magnetic structure can be described with the only AFM vector $\mathbf{L}$ with the fixed modulus. Convenient parametrization for rotation of this vector includes only two independent variables. However, times derivative, $\dot{\mathbf{L}}=\boldsymbol{\Omega} \times \mathbf{L}$, is expressed through the rotation vector. This means that for the tensors $\hat{\chi}, \hat{\alpha}$ and $\hat{g}$ the component parallel to $\mathbf{L}$ is zero, e.g. (compare with Eq. (4)):

$$
\hat{\chi}=\chi_{\perp}\left(\hat{1}-\mathbf{e}_{\mathbf{L}} \otimes \mathbf{e}_{\mathbf{L}}\right), \quad \mathbf{e}_{\mathbf{L}} \equiv \frac{\mathbf{L}}{|\mathbf{L}|} .
$$

The kinetic energy and Rayleigh function take a form:

$$
\begin{aligned}
T_{\mathrm{kin}} & =\frac{\chi_{\perp}}{2 \gamma^{2}}\left\{(\boldsymbol{\Omega}+\gamma \mathbf{H})^{2}-\left[(\boldsymbol{\Omega}+\gamma \mathbf{H}) \cdot \mathbf{e}_{\mathbf{L}}\right]^{2}\right\}, \\
\mathcal{R}_{\mathrm{AFM}} & =\frac{\alpha_{\perp}}{2 \gamma}\left[\boldsymbol{\Omega}^{2}-\left(\boldsymbol{\Omega} \cdot \mathbf{e}_{\mathbf{L}}\right)^{2}\right]-\frac{g_{\perp} j}{\gamma}\left(\boldsymbol{\Omega} \cdot \mathbf{p}_{\mathrm{curr}}\right) \\
& +\frac{g_{\perp} j}{\gamma}\left(\boldsymbol{\Omega} \cdot \mathbf{e}_{\mathbf{L}}\right)\left(\mathbf{e}_{\mathbf{L}} \cdot \mathbf{p}_{\mathrm{curr}}\right),
\end{aligned}
$$

in correspondence with the expressions deduced in Refs. 28 and 20.

\section{CURRENT-INDUCED INSTABILITY}

To illustrate an application of the obtained expressions for the Rayleigh function, we consider small oscillations of AFM vectors in the presence of dc and ac spin-polarized current (external magnetic field $\mathbf{H}_{\text {ext }}=0$ ). We start from the simplest case of isotropic noncollinear AFM. Linearized equations for $\varphi$ components are derived 
from the Exp. (24) and (20) as follows:

$$
\begin{aligned}
\ddot{\boldsymbol{\varphi}} & +\frac{\gamma \alpha_{\mathrm{AFM}}}{\chi} \dot{\boldsymbol{\varphi}}+\gamma \beta j \mathbf{p}_{\mathrm{curr}} \times \dot{\boldsymbol{\varphi}}+\frac{\gamma}{2}\left(\beta \frac{d j}{d t}-\frac{g j}{\chi}\right) \mathbf{p}_{\mathrm{curr}} \times \boldsymbol{\varphi} \\
& +\omega_{\mathrm{AFMR}}^{2} \boldsymbol{\varphi}=\frac{\gamma}{2}\left(\frac{g j}{\chi}-\beta \frac{d j}{d t}\right) \mathbf{p}_{\mathrm{curr}} .
\end{aligned}
$$

Here we take into account an "adiabatic" torque that acts as an effective field (5) and is expressed as $\mathbf{H}_{\text {eff }}^{\text {curr }} \equiv$ $\beta j \mathbf{p}_{\text {curr }}$ (where $\beta$ is the phenomenologic constant that depends upon $s d$-exchange ), $\omega_{\text {AFMR }}$ is the 3 -times degenerated AFMR frequency in the absence of field and current.

Analysis of Eq. (30) reveals some interesting features of spin-torque phenomena in AFMs. First, the current induces small rotation (dc) or small oscillations (ac) of the magnetic sublattices around $\mathbf{p}_{\text {curr }}$ direction (see Fig. 3 a). Taken, for example, $j=j_{0} e^{i \omega t}$, one obtains from (30) for the component $\varphi_{\|}=\boldsymbol{\varphi} \cdot \mathbf{p}_{\text {curr }}$ :

$$
\varphi_{\|}=\frac{\gamma j_{0}(g-i \omega \chi \beta)}{2 \chi\left[\omega_{\mathrm{AFM}}^{2}-\omega^{2}+2 i \gamma_{\mathrm{AFM}} \omega\right]},
$$

where $2 \gamma_{\mathrm{AFM}} \equiv \gamma \alpha_{\mathrm{AFM}} / \chi$ is the half-width of AFM resonance. Current density $j_{0}$ is supposed to be rather small so, that the approximation $\varphi_{\|} \ll 1$ is valid.

It is easy to see from Eq. (31) that the ac current transfers both nonadiabatic (term with $g$ ) and adiabatic (term with $\beta$ ) spin torques to AFM layer, while dc current $(\omega=0)$ transfers nonadiabatic torque only. The ac current induces the resonance at $\omega=\omega_{\mathrm{AFM}}$. The dc current induces small deflection of AFM vectors from equilibrium direction: $\mathbf{L}_{k}-\mathbf{L}_{k}^{(0)}=2 \varphi_{\|} \mathbf{p}_{\text {curr }} \times \mathbf{L}_{k}^{(0)}$. (a)

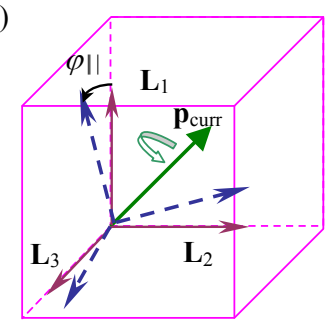

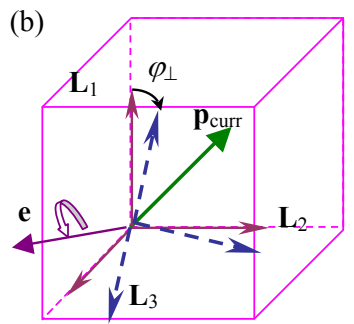

Figure 3. (Color online) Current-induced rotation of AFM structure. (a) Magnetic structure represented by three AFM vectors $\mathbf{L}_{k}$ is rotated through the fixed angle $\varphi_{\|}$ under the action of dc current. Rotation axis e (not shown) is parallel to the vector of current polarization $\mathbf{p}_{\text {curr }}$. (b) Overcritical $\left(J \geq J_{\mathrm{cr}}^{\mathrm{AFM}}\right)$ current induces instability with respect to rotation around the axis $\mathbf{e} \perp \mathbf{p}_{\text {curr }}$.
Second, nonadiabatic (Slonczewski's) contribution can induce reorientation of the magnetic structure seen as instability with respect to rotation around the axis $\mathbf{e} \perp$ $\mathbf{p}_{\text {curr }}$ (see Fig. $3 \mathrm{~b}$ ). The value of critical current at which such an instability takes place, $J_{\mathrm{cr}}^{\mathrm{AFM}}$, depends on the damping coefficient, in analogy with FM:

$$
J_{\mathrm{cr}}^{\mathrm{AFM}}=\chi \frac{4 \gamma_{\mathrm{AFM}} \omega_{\mathrm{AFMR}}}{g \gamma} S_{\mathrm{AFM}}=\chi \frac{2 \omega_{\mathrm{AFMR}}^{2}}{g \gamma Q_{\mathrm{AFM}}} S_{\mathrm{AFM}},
$$

where $Q_{\mathrm{AFM}} \equiv \omega_{\mathrm{AFMR}} /\left(2 \gamma_{\mathrm{AFM}}\right)$ is a quality factor of AFMR. However, there is substantial difference between the critical current in AFM and corresponding value for $\mathrm{FM}^{33}$ :

$$
J_{\mathrm{cr}}^{\mathrm{FM}} \propto \frac{2 \omega_{\mathrm{FMR}}^{2}}{g \gamma Q_{\mathrm{FM}}} S_{\mathrm{FM}}
$$

Really, in AFMs the critical value $J_{\mathrm{cr}}^{\mathrm{AFM}}$ is proportional to the magnetic susceptibility $\chi$ which in AFMs is rather small (compared to FM materials). So, for FMs and AFMs with equal quality factors and comparable efficiency $\epsilon_{\mathrm{s}-\mathrm{f}}$ of spin-flip scattering the critical value of current for AFMs $J_{\mathrm{cr}}^{\mathrm{AFM}} \propto J_{\mathrm{cr}}^{\mathrm{FM}} \chi \ll J_{\mathrm{cr}}^{\mathrm{FM}}$ can be significantly smaller than in FMs (due to the so called effect of the exchange enhancement).

Similar effects are predicted for the planar and collinear AFMs. The planar AFM has two different AFMR frequencies $\omega_{\mathrm{AFMR}}^{\|}<\omega_{\mathrm{AFMR}}^{\perp}$ corresponding to the in-plane and out-of-plane rotations of the magnetic vectors $\left(\omega_{\mathrm{AFMR}}^{\perp}\right.$ is double degenerated). In this case the current-induced dynamics depends upon the orientation of the current polarization vector $\mathbf{p}_{\text {curr }}$ with respect to the plane formed by AFM vectors (defined by the normal vector $\mathbf{n})$. If $\mathbf{p}_{\text {curr }} \| \mathbf{n}$, then the current induces small rotations or oscillations around $\mathbf{p}_{\text {curr }}$ with the resonant frequency $\omega_{\mathrm{AFMR}}^{\|}$(instead of $\omega_{\mathrm{AFMR}}$ in (31)), in analogy with the previous case of isotropic AFMs.

If the vector $\mathbf{p}_{\text {curr }} \perp \mathbf{n}$, then the value of the critical current at which the AFM vectors (and, correspondingly, $\mathbf{n})$ starts to rotate around $\mathbf{e} \| \mathbf{p}_{\text {curr }} \times \mathbf{n}$ (see Fig. 3 ) depends upon the magnetic anisotropy of the system, namely,

$$
J_{\mathrm{Cr}}^{\mathrm{AFM}}=\frac{2 \chi S_{\mathrm{AFM}}}{g \gamma} \sqrt{2 \gamma_{\mathrm{AFM}}^{2}\left[\left(\omega_{\mathrm{AFMR}}^{\|}\right)^{2}+\left(\omega_{\mathrm{AFMR}}^{\perp}\right)^{2}\right]+\left[\frac{\left(\omega_{\mathrm{AFMR}}^{\|}\right)^{2}-\left(\omega_{\mathrm{AFMR}}^{\perp}\right)^{2}}{4}\right]^{2}} .
$$


This result is similar to the critical current obtained for the collinear AFMs. ${ }^{20}$

It should be stressed that in contrast to FMs, the critical current is still finite even for zero dissipative constant and is dictated by magnetic anisotropy (the second term under the square root in Eq. (34)). This nontrivial effect is peculiar to AFMs and can be explained as follows. Dynamic magnetization $\mathbf{M}_{\mathrm{AFM}}$, as seen from Eq. (12), is related with the rotation of AFM vectors and hence, with circularly polarized modes of magnetic excitations (we consider only the long wave modes). Moreover, for linearly polarized oscillations of AFM vectors $\mathbf{M}_{\mathrm{AFM}}=0$. So, the spin-polarized current pumps energy into circularly polarized modes and does not interact with the linear ones. On the other hand, polarization of eigen modes depends upon the magnetic anisotropy and in the case under consideration is linear. Spin-polarized current affects not only the dissipation but the values of eigen frequencies as well. At the critical current value (34)) the splitting between the eigen frequencies is removed and circularly polarized modes start to "take energy" from the current.

Thus, current-induced dynamics of AFMs has some similarities with the dynamics of FMs, but is richer even in linear approximation. Destabilization of AFM structure (with respect to solid-like rotations) takes place at the current values that could be small compared to FMs.

However, for AFMs with four or more sublattices, the dynamic equations (30) describe only three low frequency modes. Other, so-called exchange (usually high frequency) modes are related with the mutual tilt of magnetic moments and could not be described within the present approach.

\section{DETECTION OF SPIN TRANSFER TORQUE AND SPIN-DIODE EFFECT}

Up to date the possibility to measure directly STT effects in AFMs is under discussion. Standard technique for detection of STT phenomena in the systems with active FM layer is measuring of magnetoresistance which depends upon mutual orientation of magnetizations in soft and hard layers. In this section we argue that the same approach can, in principle, be applied to the systems with the soft AFM layer.

It follows from (12) and (31) that current-induced rotation of AFM vectors produces oscillating macroscopic magnetization:

$$
\mathbf{M}_{\mathrm{AFM}}=\frac{\omega j_{0} \sqrt{g^{2}+\chi^{2} \beta^{2} \omega^{2}}}{2 \sqrt{\left(\omega_{\mathrm{AFMR}}^{2}-\omega^{2}\right)^{2}+4 \gamma_{\mathrm{AFM}}^{2} \omega^{2}}} \cos (\omega t+\phi) \mathbf{p}_{\mathrm{curr}} .
$$

where

$$
\phi \equiv \arctan \frac{g\left(\omega_{\mathrm{AFMR}}^{2}-\omega^{2}\right)-\omega^{2} \chi \beta \gamma_{\mathrm{AFM}}}{\omega\left[2 g \gamma_{\mathrm{AFM}}+\chi \beta\left(\omega_{0}^{2}-\omega^{2}\right)\right]}
$$

is the phase shift between the current and magnetization.
On the other hand, anisotropic magnetoresistance (AMR) of the multilayer, $\Delta R_{\mathrm{AMR}}$ depends upon the mutual orientation of oscillating vector, $\mathbf{M}_{\mathrm{AFM}}(t)$, and fixed magnetization of FM layer, $\mathbf{M}_{\mathrm{pol}} \| \mathbf{p}_{\mathrm{curr}}$ :

$$
\Delta R_{\mathrm{AMR}} \propto \mathbf{M}_{\mathrm{AFM}} \cdot \mathbf{p}_{\mathrm{curr}} \propto j_{0} \cos (\omega t+\phi) .
$$

This means that:

- AMR itself can be used for detection of spin-torque effect in AFM layer. In the case of multilayers with the magnetic tunnel junction (instead of metallic nonmagnetic layer) $\Delta R_{\mathrm{AMR}}$ can be as large as 130 $\%$ (see, e.g. Ref. 12);

- AMR oscillating with the same frequency as ac current can cause frequency mixing and a directly measurable dc voltage:

$$
V_{\mathrm{dc}}=\left\langle\Delta R_{\mathrm{AMR}} J\right\rangle \propto \cos \phi
$$

An analogous spin-diode effect in spin-valves with FM soft layer was already detected in Refs. 47 and 48 ;

- The abovementioned frequency mixing can reveal itself in the second-harmonic generation with corresponding voltage given by:

$$
\begin{aligned}
V_{\text {s.h. }} & =\Delta R_{\mathrm{AMR}} J \\
& \propto \frac{\omega j_{0}^{2} \sqrt{g^{2}+\chi^{2} \beta^{2} \omega^{2}}}{4 \sqrt{\left(\omega_{\mathrm{AFM}}^{2}-\omega^{2}\right)^{2}+4 \gamma_{\mathrm{AFM}}^{2} \omega^{2}}} \cos (2 \omega t+\phi) .
\end{aligned}
$$

In addition, frequency dependence of $V(\omega)$ can be used for measuring of the ratio between the dissipative (constant $g$ ) and nondissipative (constant $\beta$ ) torques in AFM. Really, in the vicinity of AFMR $\left(\left|\omega-\omega_{\mathrm{AFMR}}\right| \ll \omega_{\mathrm{AFMR}}\right)$, as follows from Eqs. (37), (38), and (39),

$$
\begin{aligned}
V_{\mathrm{dc}} & \propto \frac{1}{\sqrt{1+\omega^{2}(\chi \beta / g)^{2}}}, \\
V_{\text {s.h. }} & \propto \sqrt{1+\omega^{2}(\chi \beta / g)^{2}} .
\end{aligned}
$$

Thus, AMR and voltage are observables that provide a direct probe of the amplitude and phase of the precession of AFM vectors with respect to the ac current and make it possible to detect current-induced phenomena in AFM layer.

Expression (38) shows that spin-diode effect can be observed in the systems where an AFM plays a role of the soft magnetic layer. In contrast to FM, where spindiode effect is observed in $\mathrm{GHz}$ range, ${ }^{47}$ AFM layer can rectify the current in a higher frequency range (up to $\mathrm{THz}$ which corresponds to AFM resonance). 


\section{DISCUSSION AND CONCLUSIONS}

In the present paper we have developed a general phenomenological theory of current-induced dynamics for AFM with strong exchange coupling and have derived an expression (19) for the Rayleigh function in the presence of spin-polarized current. We have shown that spinpolarized current can produce a work over an AFM despite of the absence of equilibrium macroscopic magnetization. So, spin-polarized current can induce rotation of the magnetic moments not only in FM, but in any material which shows magnetic ordering of the exchange nature. The characteristic values of critical current depend on the efficiency of spin-flip processes at AFM/NM/FM interfaces but do not depend on the value of macroscopic magnetization of the soft layer.

Expression (19), in fact, defines the power of the external current-induced force that acts on the magnetic system at a given value of the magnetic flux $\boldsymbol{\Phi}=\nabla \cdot \hat{\boldsymbol{\Pi}} \propto$ $j \mathbf{p}_{\text {curr }}$ flowing into AFM layer (see Eq. (3)). It can be extended to the case of inhomogeneous distribution of AFM vectors (e.g., in the domain wall) in the rpesence of steady current as follows:

$$
\begin{aligned}
\mathcal{R}_{\mathrm{AFM}} & =\ldots-\int \nabla \cdot \hat{\boldsymbol{\Pi}} \cdot \boldsymbol{\Omega} d \mathbf{r} \\
& =\ldots+\frac{d}{d t} \int \hat{\boldsymbol{\Pi}}:(\nabla \otimes \boldsymbol{\theta}) d \mathbf{r} \\
& =\ldots+\frac{d}{d t} \int \Pi_{l k} \nabla_{l} \theta_{k} d \mathbf{r} \\
& =\ldots+\frac{d}{d t} \int \Pi_{l k} \delta \theta_{k} n_{l} d S .
\end{aligned}
$$

Here we denoted with ... the terms that do not depend on current.

The last equality in Eq. (41) shows that the magnetic flux density flowing in $\mathbf{n}$ direction, $n_{l} \Pi_{l k}$, and the rotational vector $\boldsymbol{\theta}$ that defines the deflection from the initial (current-free) state are thermodynamically conjugated variables. Corresponding elementary work produced by the spin polarized current at fixed $\nabla \cdot \hat{\boldsymbol{\Pi}}$ is $\delta A=\mathbf{n} \cdot \hat{\boldsymbol{\Pi}} \delta \boldsymbol{\theta}$. This means that in general, a steady current-transferred magnetic flux can change equilibrium orientation of AFM vectors (see, e.g., Eq. (31) for the case of dc current). Inversely, variation of AFM vectors (e.g. in the domain wall) should give rise to variation of the magnetic flux $\delta \hat{\boldsymbol{\Pi}}$. This, in turn, means that the spin-flux transferred by the current through the AFM domain wall or other area with inhomogeneous distribution of AFM vectors will be changed due to, e.g. depolarization. This effect can be, in principle, detected experimentally, e.g., by measuring magnetoresistance of FM/AFM/FM multilayer.

Finally, the above approach combined with the principles of nonequilibrium thermodynamics makes it possible to derive a general expression for the current-induced non-adiabatic STT for the bulk conducting AFMs. In order to demonstrate this fact we consider inhomogeneous distribution of AFM vectors described by the space dependent rotations $\varphi(\mathbf{r})$ with respect to some reference state. Then, the vector $\Omega$ is a generalized flux which is generated by thermodynamic variable $\boldsymbol{\varphi}$. Corresponding generalized forces could be obtained from the dynamic equation (see Eq. (11) added with the magnetic damping) for the electrically open AFM layer in the overdamped regime $(\dot{\boldsymbol{\Omega}} \rightarrow 0)$ :

$$
\alpha_{\mathrm{AFM}} \boldsymbol{\Omega}-\mathbf{T}_{\mathrm{AFM}}=0,
$$

where the torque $\mathbf{T}_{\mathrm{AFM}}$ is defined by the magnetic anisotropy of AFM layer (see Eq. (14) and explanation below).

The charge current density $\mathbf{j}$ injected into AFM and the electric field $\mathbf{E}$ are the other pair of the conjugated generalized thermodynamic variables. According to the Onsager principles, generalized fluxes $\boldsymbol{\Omega}, \mathbf{j}$ and generalized forces $\mathbf{T}_{\mathrm{AFM}}, \mathbf{E}$ are related as follows:

$$
\boldsymbol{\Omega}=\hat{L}_{l l} \mathbf{T}_{\mathrm{AFM}}+\hat{L}_{l q} \mathbf{E}, \quad \mathbf{j}=\hat{L}_{q l} \mathbf{T}_{\mathrm{AFM}}+\hat{L}_{q q} \mathbf{E},
$$

where the phenomenological Onsager's coefficients $\hat{L}_{\delta \xi}$, $\delta \xi=l, q$ should satisfy the rotational symmetry requirements and reciprocity relations. In assumption of the electrically homogeneous medium, $\hat{L}_{q q}=\sigma \hat{I}$, where $\sigma$ is conductivity, $\hat{I}$ is a unit matrix, and $\hat{L}_{q l}=\eta \nabla \otimes \boldsymbol{\theta}$, where $\eta$ is a material constant. From Eq. (42) it also follows that $\hat{L}_{l l}=\hat{I} / \alpha_{\mathrm{AFM}}$

With account of time-inversion symmetry of AFM the reciprocity relations state that $\hat{L}_{q l}=-\hat{L}_{l q}$ if all the equilibrium magnetic vectors of AFM change sign under the time reversal.

Thus, after quite simple mathematics we get from Eq. (43)

$$
\boldsymbol{\Omega}=\frac{1}{\alpha_{\mathrm{AFM}}} \mathbf{T}_{\mathrm{AFM}}-\frac{\eta}{\sigma}(\mathbf{j}, \nabla) \boldsymbol{\theta} .
$$

Last term in the expression (44) generalizes the expression for the dissipative torque obtained in Ref. 49 (Eq. (9) there) for a particular case of a collinear AFM. We stress that this effect, in contrast to those considered above, reflects the action of AFM (as potential polarizer) on the non-polarized current.

Current-induced STT in AFMs can be used for the description of current-induced dynamics of the domain walls and other inhomogeneous states in the conducting AFMs and other materials with the nontrivial magnetic structure. It should be noted that thus introduced dissipative torque accounts for the rotation (in space or in time) of the magnetic sublattices only and does not take into account flip processes (current-induced transition from AFM to FM structure) that could take place at the substantially higher current density.

The last question that is worth to discuss here is the effect of the ac current that results from the direct $s d$ exchange between free and localized electrons (the effective magnetic field $\mathbf{H}_{\text {eff }}^{\text {curr }}$, Eq. (5)). As it follows from the 
dynamic Eqs. (30), (31), time-derivative dj/dt of spinpolarized current works as a driving force for oscillations of AFM moments. Its value growth linearly with the current frequency. This effect has no counterpart in FM, where the analogous field-like torque is proportional to $j$, but not to $d j / d t$. Thus, in AFM the current-induced movements of AFM vectors are controlled by two "forces" which are $90^{\circ}$ shifted in phase. The phase shift between the current and system response depends upon the frequency. This opens an additional way for experimental observation of current-induced phenomena in AFMs.

E.V.G. is grateful B. Ivanov for valuable discussion. The authors acknowledge the financial support from the Physics and Astronomy Department of National Academy of Science of Ukraine in the framework of the Special Program for Fundamental Researches. The work was partially supported by the grant from Ministry of Education and Science of Ukraine.

Appendix A: Description of macroscopic magnetic dynamics with the help of the Gibbs' vector

The Gibbs' vector $\boldsymbol{\varphi}$ gives a convenient way for parametrization of the rotational symmetry group $O(3)$. Group multiplication (symbol o) of two rotations, $\varphi_{1}$ and than $\boldsymbol{\varphi}_{2}$, is noncommutative and is given by the rule:

$$
\varphi_{2} \circ \varphi_{1} \equiv \frac{\varphi_{2}+\varphi_{1}+\left[\varphi_{2} \times \varphi_{1}\right]}{1-\varphi_{2} \varphi_{1}}
$$

In application to the description of macroscopic magnetic dynamics the vector $\varphi$ is a field variable that characterizes the state of magnetic system at a time moment $t$ and in a space point $\mathbf{r}$ respective to some reference (equilibrium) state. "Radius-vector" $\delta \boldsymbol{\theta} / 2$ between two states at different moments, $\boldsymbol{\varphi}(t)$ and $\boldsymbol{\varphi}(t+d t)=\boldsymbol{\varphi}(t)+d \boldsymbol{\varphi}$ is given by Eq. (6) and can be found from Eq. (A1) if we set

$$
\frac{\delta \boldsymbol{\theta}}{2}=[\boldsymbol{\varphi}(t)+d \boldsymbol{\varphi}] \circ[-\boldsymbol{\varphi}(t)] .
$$

"Radius-vector" between two states in different points $\mathbf{r}$ and $\mathbf{r}+d \mathbf{r}$ is found in a similar way.

Equilibrium magnetic structure of any AFM with strong exchange coupling can be described with the use of up to three orthogonal vectors, $\mathbf{L}_{k}^{(0)}(k=1,2,3)$. Once $\mathbf{L}_{k}^{(0)}$ are known, orientation of AFM vectors at any $t$ and $\mathbf{r}, \mathbf{L}_{k}(t, \mathbf{r})$, can be expressed in terms of the Gibbs's vector as follows:

$$
\mathbf{L}_{k}=\mathbf{L}_{k}^{(0)}+\frac{2}{1+\varphi^{2}}\left[\left[\boldsymbol{\varphi} \times\left[\boldsymbol{\varphi} \times \mathbf{L}_{k}^{(0)}\right]\right]+\left[\boldsymbol{\varphi} \times \mathbf{L}_{k}^{(0)}\right]\right] .
$$

The potential energy $U_{\mathrm{AFM}}(\boldsymbol{\varphi})$ in Eq. (9) can be found in a following way. One must construct a symmetryinvariant expression for $U_{\mathrm{AFM}}(\varphi)$ in terms the components of $\mathbf{L}_{k}$ vectors and then express $\mathbf{L}_{k}$ in terms of $\varphi$ using the relation (A3).
1 D. C. Ralph and M. D. Stiles, J. Magn. Magn. Mater 320, 1190 (2008)

2 L. Berger, Phys. Rev. B 54, 9353 (1996)

3 J. C. Slonczewski, Phys. Rev. B 39, 6995 (1989)

4 J. Slonczewski, J. Magn. Magn. Mater. 159, L1 (1996)

5 Z. Wei, A. Sharma, A. S. Nunez, P. M. Haney, R. A. Duine, J. Bass, A. H. MacDonald, and M. Tsoi, Phys. Rev. Lett. 98, 116603 (2007)

6 S. Urazhdin and N. Anthony, Phys. Rev. Lett. 99, 046602 (2007)

7 X.-L. Tang, H.-W. Zhang, H. Su, Z.-Y. Zhong, and Y.-L. Jing, Applied Physics Letters 91, 122504 (2007)

8 N. V. Dai, N. C. Thuan, L. V. Hong, N. X. Phuc, Y. P. Lee, S. A. Wolf, and D. N. H. Nam, Phys. Rev. B 77, 132406 (2008)

9 J. Bass, A. Sharma, Z. Wei, and M. Tsoi, J. of Magnetics (Korean Magnetics Society) 13, 1 (2008)

10 J. C. Lee, M. G. Chun, W. H. Park, C.-Y. You, S.-B. Choe, W. Y. Yung, and K. Y. Kim (AIP, 2006) p. 08G517

11 W. Park, D. V. Baxter, S. Steenwyk, I. Moraru, W. P. Pratt, and J. Bass, Phys. Rev. B 62, 1178 (2000)

12 B. G. Park, J. Wunderlich, X. Marti, V. Holy, Y. Kurosaki, M. Yamada, H. Yamamoto, A. Nishide, J. Hayakawa,
H. Takahashi, A. B. Shick, and T. Jungwirth, Nature Materials 10, 347 (2011)

13 A. B. Shick, S. Khmelevskyi, O. N. Mryasov, J. Wunderlich, and T. Jungwirth, Phys. Rev. B 81, 212409 (2010)

14 Y. Xu, S. Wang, and K. Xia, Phys. Rev. Lett. 100, 226602 (2008)

15 R. A. Duine, P. M. Haney, A. S. N. nez, and A. H. MacDonald, Phys. Rev. B 75, 014433 (2007)

16 P. M. Haney and A. H. MacDonald, Phys. Rev. Lett. 100, 196801 (2008)

17 K. M. D. Hals, Y. Tserkovnyak, and A. Brataas, Phys. Rev. Lett. 106, 107206 (2011)

18 A. Manchon, N. Ryzhanova, A. Vedyayev, and B. Dieny, J. Appl. Phys. 103, 070000 (2008)

19 E. V. Gomonay and V. M. Loktev, Low Temperature Physics 34, 198 (2008)

20 H. V. Gomonay and V. M. Loktev, Phys. Rev. B 81, 144427 (2010)

21 Y. V. Gulyaev, P. E. Zilberman, and E. M. Epshtein, ArXiv e-prints(Nov. 2010), arXiv:1011.4303

22 A. F. Andreev and V. I. Marchenko, Physics-Uspekhi 23, $21(1980)$

23 A. F. Andreev and V. I. Marchenko, JETP 43, 794 (1976) 
24 We exclude any possible solid-like rotation of the crystal lattice assuming that the sample is fixed and consider rotation of the magnetic subsystem only. However, STT can, in principle, give rise to rotation of the crystal lattice and/or to the combined magneto-mechanical oscillations, due to the spin-lattice coupling. These problems though interesting are out of scope of this paper.

25 J. C. and Slonczewski, J. Magn. Magn. Mat. 195, L261 (1999)

26 In many textbooks and papers the modulus of gyromagnetic ratio is used, this give rise to opposite sign before the second term in (2)

27 H. Kohno and J. Shibata, J. Phys. Soc. Japan 76, 063710 (2007)

28 I. Bar'yakhtar and B. Ivanov, Sov. J. Low Temp. Phys. 5, $361(1979)$

29 S. Zhang and Z. Li, Phys. Rev. Lett. 93, 127204 (2004)

30 A. V. Kimel, B. A. Ivanov, R. V. Pisarev, P. A. Usachev, A. Kirilyuk, and T. Rasing, Nature Physics 5, 727 (2009)

31 T. Satoh, S. Cho, R. Iida, T. Shimura, K. Kuroda, H. Ueda, Y. Ueda, B. A. Ivanov, F. Nori, and M. Fiebig, Phys. Rev. Lett. 105, 077402 (Aug 2010)

32 Full time derivative includes $\dot{\varphi}(\partial / \partial \varphi)$.

33 B. A. Ivanov and C. E. Zaspel, Phys. Rev. Lett. 99, 247208 (2007)

34 Y. Endoh and Y. Ishikawa, J. Phys. Soc. Japan 30, 1614 (1971)

${ }^{35}$ R. Jungblut, R. Coehoorn, M. T. Johnson, J. aan de Stegge, and A. Reinders, J. Appl. Phys. 75, 6659 (1994)

${ }^{36}$ F. Offi, W. Kuch, and J. Kirschner, Phys. Rev. B 66, 064419 (2002)

37 G. M. Stocks, W. A. Shelton, T. C. Schulthess, B. Uj- falussy, W. H. Butler, and A. Canning, J. Appl. Phys. 91 , 7355 (2002)

38 Disordered magnetics also include FM materials with nonzero magnetization, however, here we are interested only in materials with zero or small macroscopic magnetic moment.

39 L. Szunyogh, B. Lazarovits, L. Udvardi, J. Jackson, and U. Nowak, Phys. Rev. B 79, 020403(R) (2009)

40 R. Acharyya, H. Yen Thi Nguyen, W. P. Pratt, Jr., and J. Bass, APS Meeting Abstracts, 36001(Mar. 2010)

41 J. P. King, J. N. Chapman, M. F. Gillies, and J. C. S. Kools, J. Phys. D: Appl. Phys. 34, 528 (2001)

42 D. Fruchart, E. F. Bertaut, R. Madar, G. Lorthioir, and R. Fruchart, Solid State Comm. 9, 1793 (1971)

${ }^{43}$ K. Takenaka and H. Takagi, Appl. Phys. Lett. 87, 261902 (2005)

44 K. Takenaka and H. Takagi, Materials transactions 47, 471 (2006)

45 K. Kodama, S. Iikubo, K. Takenaka, M. Takigawa, H. Takagi, and S. Shamoto, Phys. Rev. B 81, 224419 (2010)

46 K. Kamishima, T. Goto, H. Nakagawa, N. Miura, M. Ohashi, N. Mori, T. Sasaki, and T. Kanomata, Phys. Rev. B 63, 024426 (2000)

47 A. A. Tulapurkar, Y. Suzuki, A. Fukushima, H. Kubota, H. Maehara, K. Tsunekawa, D. D. Djayaprawira, N. Watanabe, and S. Yuasa, Nature 438, 339 (2005)

48 D. Fang, H. Kurebayashi, J. Wunderlich, K. Vyborny, L. P. Zarbo, R. P. Campion, A. Casiraghi, B. L. Gallagher, T. Jungwirth, and A. J. Ferguson, ArXiv e-prints(2010), arXiv:1012.2397

49 Y. Tserkovnyak, H. J. Skadsem, A. Brataas, and G. E. W. Bauer, Phys. Rev. B 74, 144405 (2006) 